\title{
Evaluation of Hyponatremia and Predictors of Hyponatremia in Patients Hospitalized with the COVID-19
}

\author{
(1) Emine Emektar ${ }^{1}$, (1) Fatma Nur Karaarslan¹, (1) Filiz Koç², (1) Seda Dağar ${ }^{1}$, (1) Hüseyin Uzunosmanoğlu1, \\ (1) Șeref Kerem Çorbacıoğlu1
}

${ }^{1}$ Clinic of Emergency Medicine, Keçiören Training and Research Hospital, Ankara, Turkey

${ }^{2}$ Clinic of Infectious Diseases, Keçiören Training and Research Hospital, Ankara, Turkey

\begin{abstract}
Aim: The infection caused by severe acute respiratory syndrome coronavirus 2 (SARS-CoV-2), called coronavirus disease-2019 (COVID-19), has been declared by the World Health Organization as a pandemic. Moderate and severe hyponatremia may develop in COVID-19 cases. This study aimed to evaluate the frequency and predictors of hyponatremia in patients hospitalized with COVID-19.

Materials and Methods: This retrospective study included patients aged $>18$ years who were admitted to our hospital between August $1^{\text {st }}$, 2020, and September $30^{\text {th }}, 2020$, with positive RNA polymerase chain reaction (PCR) test. Patients' demographic data, comorbidities, vital signs, physical examination findings, laboratory results, sodium levels, and hospital outcomes were evaluated.

Results: The study included 760 patients with positive PCR test results. Of all the patients, $47.6 \%$ were female, and the median age was 63 years. The in-hospital mortality rate was $11.7 \%$. The median sodium level was 137 (134-140). Hyponatremia was present in $25.7 \%$ of the patients, and majority of the patients had mild hyponatremia. Hyponatremia was observed more frequently in patients with advanced age, male gender, diabetes, and severe pneumonia ( $p=0.041, p=0.003, p<0.001, p=0.002$, respectively).

Conclusion: In this study, hyponatremia is relatively common in patients admitted with COVID-19 pneumonia, and it is associated with higher disease severity. The incidence of hyponatremia increases in older patients, male patients, patients with severe pneumonia, and patients with diabetes.
\end{abstract}

Keywords: COVID-19, hyponatremia, pneumonia

\section{Introduction}

The severe acute respiratory syndrome coronavirus 2 (SARSCoV-2) infection epidemic has been named coronavirus disease 2019 (COVID-19) by the World Health Organization (WHO). COVID-19 disease is a respiratory disease detected in Wuhan, China, caused by a new coronavirus. COVID-19 spread rapidly to many countries and was declared as a pandemic by WHO (1). The disease is highly contagious and its main clinical symptoms are fever, dry cough, fatigue, myalgia, and shortness of breath. However, all these symptoms are not specific to the disease and the disease can progress rapidly to severe pneumonia. Severe acute respiratory syndrome (SARS) may develop in more severe cases. Pathogenesis of coronavirus-associated SARS is associated with cytokine storm that involves high serum proinflammatory cytokines and chemokines [interleukins 1, 6, 8 and 12 (IL1, IL-6, IL-8, IL-12), tumor necrosis factor (TNF) - alpha and interferon (IF-N) - gamma] (2). A serum sodium concentration below $135 \mathrm{mmol} / \mathrm{L}$ is called hyponatremia. Hyponatremia is the most common electrolyte disorder. Hyponatremia is found in approximately $10 \%-15 \%$ of hospitalized patients (3). One of the common causes of hyponatremia is Syndrome of inappropriate antidiuretic hormone syndrome (SIADH) due to lung diseases (4). It occurs in approximately $25 \%$ of patients with pneumonia $(3,4)$. In patients with pneumonia, a relationship between the presence of hyponatremia with a prolonged hospital stay, increased risk 
of admission to the intensive care unit (ICU), high hospital costs, and increased mortality has been observed (4-6). In patients with severe hyponatremia, it can be seen life-threatening complications such as encephalopathy, cerebral edema, seizures, and coma. Although the risk of hyponatremia among pneumonia patients varies according to the pathogen, $44 \%-46 \%$ of patients with Legionella pneumonia develop hyponatremia, and hyponatremia is observed in $8 \%-14 \%$ of patients in other community-acquired pneumonia etiologies $(7,8)$.

In recent studies and case reports, it has been reported that patients with SARS-CoV-2 infection may develop moderate and severe hyponatremia (9-11). In these studies, there are publications suggesting that the presence of hyponatremia during the admission to hospital may be an indicator of potential COVID-19 infection (11). It is now known that severe systemic inflammation develops in COVID-19 patients. It is also thought that systemic inflammation may cause hyponatremia due to non-osmotic stimulation for antidiuretic hormone production. However, data on the prevalence, severity, and impact of hyponatremia in patients with COVID-19 are limited in the literature.

In this study, we aimed to evaluate the frequency of hyponatremia and predictors of hyponatremia in patients hospitalized due to COVID-19.

\section{Materials and Methods}

The study is retrospective and observational. Prior to implementation, our study's protocol was approved by the Ethics Committee of Ankara Keçiören Training and Research Hospital (2012-KAEK-15/2187) and Ministry of Health Scientific Research Committee. Since the first case of COVID-19 detected in our country, hospitalization, diagnosis, and treatment of patients are planned in our hospital according to the national COVID-19 guidelines published by the Ministry of Health. Our hospital has been serving as a pandemic hospital since April 2020. Patients are evaluated in emergency department and pandemic outpatient clinics and laboratory (complete blood count, routine biochemical tests, C-reactive protein, erythrocyte sedimentation rate, prothrombin time, partial thromboplastin time, D-dimer, fibrinogen). ferritin, and imaging studies [postero-anterior chest radiography and/or low-dose computed tomography (CT) of the thorax without contrast] are required.

Patients over the age of 18 who were admitted to Keçiören Training and Research Hospital between 01.08.2020-30.09.2020 with positive RNA polymerase chain reaction (PCR) test results that were followed up by hospitalization were included in the study.
Demographic data, complaints, comorbid diseases, vital signs, physical examination findings, laboratory results, hospital stay, and hospital outcomes of the patients were obtained from the hospital automation system and patient files. Hyponatremia was defined by natremia below $135 \mathrm{mmol} / \mathrm{L}$. Patients were biochemically divided into subgroups according to plasma sodium concentrations at the time of admission to emergency department: mild hyponatremia (130-134 mmol/L), moderate hyponatremia (125-129 mmol/L), and severe hyponatremia (<125 mmol/L) (10). Patients referred from another hospital and who were found to have pseudo-hyponatremia as a result of etiological evaluation were excluded from the study. Patients who developed hyponatremia during their hospitalization were not included in the study, since iatrogenic causes of hyponatremia would confound analysis of the effect of SARSCoV-2 on sodium levels. Patients were divided into two groups according to thorax CT. Patients with more than $50 \%$ of the entire lung area affected were considered to have moderate to severe pneumonia accordance with the national COVID-19 guidelines published by the Ministry of Health. In-hospital deaths of the patients were recorded.

Patients with incomplete data and those with negative PCR test results who were followed up with a pre-diagnosis of COVID-19 were excluded from the study.

\section{Statistical Analysis}

The analysis of all data obtained during the study and recorded in the study form was performed using IBM SPSS 20.0 (Chicago, IL, USA) statistics program. Whether the distribution of discrete and continuous numerical variables is suitable for normal distribution was investigated by the Kolmogorov-Smirnov test. Descriptive statistics and discrete and continuous numerical variables were shown as median [interquartile range (IQR): 25-75], and categorical variables as a number of cases and (\%). Categorical variables were evaluated with chi-square and continuous variables with the Mann-Whitney U test. Predictors of hyponatremia were determined using univariate tests, and statistically significant $(p<0.2)$ variables were tested with a multivariate logistic regression model. The fitness of this model was tested with the Hosmer-Lemeshow test. Before being included in the model, the correlation of the data was evaluated using the Spearman correlation test. $p<0.05$ were considered statistically significant.

\section{Results}

During the study period, 1,089 patients were hospitalized with the pre-diagnosis of COVID-19, and 760 patients with positive PCR test and complete data were included in the study (19 
patients were excluded because of missing data, 12 patients were excluded because of hypernatremia, 308 patients were excluded because of the PCR test is negative or missing).

$47.6 \%$ of the patients were female and the median age was 63 (IQR: 52-72). The most common comorbidity in patients was hypertension with $43.9 \%$. It was observed that the most common complaints of the patients were fever (61.2\%) and cough (55.3\%). Pneumonia was present in $89.7 \%$ of the patients and the lesions were bilateral in $84.5 \%$ of the patients. Eighty-nine (11.7\%) patients died. The demographic data of the patients are given in Table 1.

Laboratory parameters measured at the time of admission are given in Table 2. The median sodium value of the patients was 137 (134-140). Hyponatremia was present in $25.7 \%$ of the patients, and the majority of the patients had mild hyponatremia (Table 3).

When hyponatremic and normonatremic patients were compared; It was determined that hyponatremic patients were older, hypertension, diabetes, coronary artery disease was more common, their saturations measured the time of admission were lower, they had more pneumonic infiltration and severe pneumonia in chest imaging, and these patients needed more mechanical ventilation (for all values, $p<0.05$ ). In addition, patients with hyponatremia were found to have higher mortality and longer hospital stay (for all values, $p<0.05$ ) (Table 4).

Multivariate logistic regression analysis was performed to examine the effects of variables examined in Table 4 on hyponatremia together with other variables. Firstly, correlation analyses were made. It was observed that mechanical ventilation need correlated with (ICU) need, pneumonia correlated the severity of pneumonia, pneumonia correlated saturation and ICU need, and pulse rate/fever correlated with saturation $(p<0.05$, $r>0.3$ ). Diabetes, hypertension, saturation at admission, ICU need, severe pneumonia, time from onset to hospitalization, age, and gender were included in the multivariate model because of a p-value equal to or below 0.2 from Table 4 and noncorrelated or weak correlated in correlation analysis $(p>0.05, p<0.05$ and $r<0.3$ )

After the model established by the Hosmer-Lemeshow test was found to be fit, it was found that hyponatremia was observed more frequently in patients with advanced age, male gender, diabetes, and severe pneumonia ( $p=0.041, p=0.003, p<0.001$, $p=0.002$, respectively) (Table 5).

\begin{tabular}{|c|c|}
\hline Age, years, median IQR (25-75) & $63(52-72)$ \\
\hline \multicolumn{2}{|l|}{ Sex, n (\%) } \\
\hline Female & $362(47.6 \%)$ \\
\hline \multicolumn{2}{|l|}{ Comorbidity, n (\%) } \\
\hline Hypertension & $334(43.9 \%)$ \\
\hline Diabetes & $224(29.5 \%)$ \\
\hline Coronary heart disease/KKY & $132(17.4 \%)$ \\
\hline Chronic obstructive lung disease & $59(7.8 \%)$ \\
\hline Hyperthyroidism & $48(6.3 \%)$ \\
\hline Chronic kidney disease & $36(4.7 \%)$ \\
\hline Other & $77(10.1 \%)$ \\
\hline \multicolumn{2}{|l|}{ Drugs, n (\%) } \\
\hline Angiotensin-converting enzyme inhibitor & $132(17.4 \%)$ \\
\hline Angiotensin receptor blocker & $79(10.4 \%)$ \\
\hline Diuretic & $113(14.9 \%)$ \\
\hline \multicolumn{2}{|l|}{ Vital signs, median IQR (25-75) } \\
\hline Respiratory rate & $16(14-18)$ \\
\hline Pulse & $75(70-82)$ \\
\hline Body temperature & $37.6(37-38.1)$ \\
\hline Saturation & $90(86.2-92)$ \\
\hline \multicolumn{2}{|l|}{ *Admission complaints of patients, $\mathbf{n}(\%)$} \\
\hline Fever & $465(61.2 \%)$ \\
\hline Cough/sputum & $420(55.3 \%)$ \\
\hline Dyspnea & $349(45.9 \%)$ \\
\hline Myalgia/fatigue & $230(30.3 \%)$ \\
\hline Throat ache & $55(7.2 \%)$ \\
\hline Diarrhea & $82(10.8 \%)$ \\
\hline Nausea or vomiting & $137(18 \%)$ \\
\hline Headache & $73(9.6 \%)$ \\
\hline Anosmia & $41(5.4 \%)$ \\
\hline \multicolumn{2}{|l|}{ Pneumonia, n (\%) } \\
\hline No-pneumonia & $78(10.3 \%)$ \\
\hline \multicolumn{2}{|l|}{ Pneumonia severity } \\
\hline Mild & 487 (71.4\%) \\
\hline Moderate-severe & $195(28.6 \%)$ \\
\hline \multicolumn{2}{|l|}{ CT results, n (\%) } \\
\hline Bilateral involvement & $642(84.5 \%)$ \\
\hline $\begin{array}{l}\text { Time from illness onset to hospital admission, days, } \\
\text { median IQR (25-75) }\end{array}$ & $5(3-7)$ \\
\hline \multicolumn{2}{|l|}{ Oxygen treatment, n (\%) } \\
\hline Non-invasive mechanical ventilation & $59(7.8 \%)$ \\
\hline Invasive mechanical ventilation & $74(9.7 \%)$ \\
\hline High flow oxygen treatment & $89(11.7 \%)$ \\
\hline Renal replacement treatment, n (\%) & $14(1.8 \%)$ \\
\hline \multicolumn{2}{|l|}{ Prognosis, n (\%) } \\
\hline Healed and discharged & $671(88,3 \%)$ \\
\hline Death & $89(11.7 \%)$ \\
\hline ICU hospitalization, n (\%) & $92(12.1 \%)$ \\
\hline ICU stay time, median IQR (25-75) & $13(5-25)$ \\
\hline Hospitalization length, median IQR (25-75) & $6(5-10)$ \\
\hline
\end{tabular}




\begin{tabular}{|l|l|}
\hline \multicolumn{2}{|l|}{ Table 2. Laboratory parameter of all patients upon admission } \\
\hline Median IQR (25-75) & $13.3(12.2-14.5)$ \\
\hline Hemoglobin & $6.300(4.7-8.5)$ \\
\hline White blood cell & $4.66(3.14-6.98)$ \\
\hline Neutrophil & $1.05(0.72-1.49)$ \\
\hline Lymphocyte & $208(159-272)$ \\
\hline Platelet & $53.4(17.2-115.9)$ \\
\hline C-reactive protein & $53(34-74)$ \\
\hline Sedimentation & $302.7(132.3-646)$ \\
\hline ferritin & $640(370-1142.5)$ \\
\hline D dimer & $509.2(409-631)$ \\
\hline Fibrinogen & $124(103-169)$ \\
\hline Glucose & $53(34-474)$ \\
\hline Urea & $1(0.835-1.19)$ \\
\hline Creatinine & $72.3(55.2-87.8)$ \\
\hline Glomerular filtration rate & $3.5(3.2-3.8)$ \\
\hline Albumin & $137(134-140)$ \\
\hline Sodium & $4.2(3.94-4.55)$ \\
\hline Potassium & $101.5(98-104)$ \\
\hline Chlorine & $5.15(2.5-14.3)$ \\
\hline Troponin & \\
\hline
\end{tabular}

\section{Discussion}

In this study, in which we evaluated the frequency of hyponatremia and its relation with mortality in patients hospitalized for COVID-19, we first showed that hyponatremia was common in COVID-19 patients, and approximately $25 \%$ of patients have hyponatremia. Secondly, we found that patients with hyponatremia were older, had more comorbidity, more severe pneumonia, and more need for mechanical ventilation, longer hospital stay, and more mortality. Finally, we found that hyponatremia was more common in older age, male gender, and those with diabetes and severe pneumonia.

\begin{tabular}{|l|l|}
\hline \multicolumn{2}{|l|}{ Table 3. Sodium levels of all patients } \\
\hline Hyponatremia & $195(25.7 \%)$ \\
\hline Normonatremia & $565(74.3)$ \\
\hline Hyponatremia severity & \\
\hline Mild & $152(77.9 \%)$ \\
\hline Moderate & $32(16.4 \%)$ \\
\hline Severe & $11(5.6 \%)$ \\
\hline
\end{tabular}

\begin{tabular}{|c|c|c|c|}
\hline & Hyponatremia $(n=195)$ & Normonatremia $(n=565)$ & $p$-value \\
\hline Age, years, median IQR (25-75) & $65(56-73)$ & $62(50-71)$ & 0.002 \\
\hline \multicolumn{4}{|l|}{ Sex, n (\%) } \\
\hline Female & $76(39 \%)$ & $286(50.6 \%)$ & 0.005 \\
\hline \multicolumn{4}{|l|}{ Comorbidity, $\mathbf{n}(\%)$} \\
\hline Hypertension & $109(55.9 \%)$ & $225(39.8 \%)$ & $<0.001$ \\
\hline Diabetes & $88(45.1 \%)$ & $136(24.1 \%)$ & $<0.001$ \\
\hline Coronary heart disease & $44(22.6 \%)$ & $88(15.6 \%)$ & 0.029 \\
\hline Chronic obstructive lung disease & $10(5.1 \%)$ & $49(8.7 \%)$ & 0.211 \\
\hline Chronic kidney disease & $13(6.7 \%)$ & $23(4.1 \%)$ & 0.191 \\
\hline Hypothyroidism & $12(6.2 \%)$ & $36(6.4 \%)$ & 0.914 \\
\hline \multicolumn{4}{|l|}{ Vital signs, n (\%) } \\
\hline Pulse & $76(71-82)$ & $75(70-81)$ & 0.187 \\
\hline Saturation & $89(83-91)$ & $90(88-92)$ & $<0.001$ \\
\hline Fever (temperature) & $37.8(37-38.2)$ & $37.6(37-38)$ & 0.07 \\
\hline Pneumonia Presence, $\mathrm{n}(\%)$ & $187(95.9 \%)$ & $495(87.6 \%)$ & 0.001 \\
\hline Severe pneumonia, n (\%) & $75(38.5 \%)$ & $120(21.2 \%)$ & $<0.001$ \\
\hline The need for mechanical ventilation, $\mathrm{n}(\%)$ & $27(13.8 \%)$ & $47(8.3 \%)$ & 0.025 \\
\hline ICU need, n (\%) & $34(17.4 \%)$ & $58(10.3 \%)$ & 0.08 \\
\hline \multicolumn{4}{|l|}{ CT results, $\mathbf{n}(\%)$} \\
\hline Bilateral involvement & $65(43.3 \%)$ & $7(38.9 \%)$ & 0.719 \\
\hline $\begin{array}{l}\text { Time from illness onset to } \\
\text { hospital admission, days, median IQR (25-75) }\end{array}$ & $5(4-7)$ & $5(3-7)$ & 0.065 \\
\hline Hospitalization length, median IQR (25-75) & $7(5-12)$ & $6(5-9)$ & 0.015 \\
\hline ICU time, median IQR (25-75) & $12(8-22)$ & $13.5(5-28)$ & 0.713 \\
\hline In hospital mortality, n (\%) & $35(25.7 \%)$ & $54(9.6 \%)$ & 0.002 \\
\hline
\end{tabular}




\begin{tabular}{|c|c|c|c|c|}
\hline & Wald & p-value & OR & $95 \% \mathrm{Cl}$ \\
\hline Age & 4.165 & 0.041 & 0.98 & 0.97-0.99 \\
\hline Diabetes & 21.472 & $<0.001$ & 2.36 & $1.64-3.4$ \\
\hline Hypertension & 1.941 & 0.164 & 1.31 & $0.89-1.93$ \\
\hline $\begin{array}{l}\text { Time from illness onset to } \\
\text { hospital admission, days }\end{array}$ & 0.085 & 0.770 & 0.98 & $0.91-1.06$ \\
\hline ICU need & 0.009 & 0.924 & 0.97 & $0.547-1.72$ \\
\hline Severe pneumonia & 9.834 & 0.002 & 1.93 & $1.28-2.93$ \\
\hline
\end{tabular}

Hyponatremia is most common electrolyte disorder in the clinic. Hyponatremia can be classified in various ways according to its severity (12). According to the blood levels, it can be classified biochemically as mild, moderate, and severe; according to the rate of development, those that develop in the last 48 hours are classified as acute hyponatremia, and those developing longer than 48 hours as chronic hyponatremia. Mild hyponatremia was present in most of our patients (77.9\%) with hyponatremia. Hyponatremia is seen in $26 \%-28 \%$ of patients with pneumonia $(4,13)$, and it has been observed that it is associated with an increased risk of ICU hospitalization, long-term stay, higher hospital costs, and increased mortality rates $(4,6,7)$. Severe hyponatremia is associated with life-threatening complications, including encephalopathy, cerebral edema, seizures, coma, and pontine myelinolysis (secondary to increase excessively rapid sodium correction) (12). The frequency of developing hyponatremia among pneumonia patients varies. In studies published recently in the literature, moderate and severe hyponatremia has been reported in patients with SARS-CoV-2 infection $(9,10)$. They suggested that the hyponatremia detected at admission could be an indicator of potential COVID-19 infection (11).

The frequency and severity of hyponatremia increase with age $(12,14)$. In a prospective study, it was shown that the sodium level of $1 / 3$ of the elderly individuals was $<135 \mathrm{mmol} / \mathrm{L}$, and in another study, the frequency of hyponatremia was $11.6 \%$ in individuals over the age of $75(14,15)$. Hyponatremia in the elderly is one of the common causes of emergency admission (16). Hyponatremia in the elderly is often multifactorial and SIADH is the most common cause of hyponatremia (17). In addition to some diseases, many drugs can cause both direct effect and SIADH, and patients may develop hyponatremia $(17,18)$. In our study, approximately $45 \%$ of our patients consist of geriatric patients. Most of the geriatric patients have at least one comorbidity, and hyponatremia in these patients may have developed due to the above conditions together with the COVID-19 infection.

In COVID-19, hyponatremia may occur due to some physiopathological changes. It is thought that it most commonly develops secondary to pneumonia $(10,11)$. The development of hyponatremia due to pneumonia has been known for a long time. However, the mechanism of hyponatremia in pneumonia is not clearly understood. Often thought to be SIADH (10). Hypovolemia may develop in these concomitant patients due to factors such as insufficient oral intake, systemic vasodilation, vomiting, and extrarenal sodium losses due to diarrhea. In COVID-19, hyponatremia may develop due to both pulmonary and extrapulmonary mechanisms $(19,20)$.

We found that hyponatremia developed more in patients with severe pneumonia (significant involvement on $\mathrm{CT}$ ) and patients with low saturation. Mechanical ventilation and ICU requirements were higher in our patients with hyponatremia. As pneumonia severity increased in patients with COVID-19, hyponatremia was more frequent and more severe. Berni et al. (21) found a more severe outcome (a composite of disease severity which included mortality) in patients with hyponatremia hospitalized for COVID-19 in a small retrospective study.

In the regression analysis where we investigate the effects of the variables we obtained in univariate analysis in our study on hyponatremia, we found that the male gender developed hyponatremia 1.7 times, diabetes 2.3 times, and severe pneumonia 1.9 times more. Hyponatremia is an expected condition in advanced age and diabetic patients. Gender factor is an interesting result in the development of hyponatremia. Male patients came to the emergency department after a median of 5 (4-7) days after complaints start, and female patients in a median of 4 (3-6) days after the complaints start. We think that the late admission of male patients to the hospital and starting 
treatment later than women predispose to the increase in the severity of pneumonia and hyponatremia.

\section{Study Limitations}

Our study has some limitations. First of all, since it is a singlecenter study, our results cannot be generalized to all centers. Secondly, due to the retrospective nature of the study, missing data may have affected the results of the study. Also, since urinary sodium, osmolality, and Interleukin 6 levels were not examined in all patients, they were not included in the analysis. Consecutive sodium measurements were not performed in all patients. The median age of our patients is advanced and most of them have an accompanying disease. In addition, patients with COVID-19 who were discharged from the emergency department or who did not have an indication for hospitalization were excluded. Also, we were not evaluated the clinical findings and course of the patients. These situations may have affected our results.

\section{Conclusion}

Hyponatremia was common in patients admitted with COVID-19 pneumonia, and it is associated with COVID-19 disease severity in our study. The incidence of hyponatremia increases in geriatric patients, male patients, patients with severe pneumonia, and diabetic patients. The emergency physician should be aware that COVID-19 patients may have hyponatremia due to pneumonia and that the disease will be more severe in these patients, and the need for more intensive care and mechanical ventilation. Studies on larger cohorts may shed light on the physiopathology of hyponatremia in COVID-19.

\section{Ethics}

Ethics Committee Approval: The study is retrospective and observational. Prior to implementation, our study's protocol was approved by the Ethics Committee of Ankara Keçiören Training and Research Hospital (2012-KAEK-15/2187) and Ministry of Health Scientific Research Committee.

Informed Consent: This is a retrospective study.

Peer-review: Externally peer-reviewed.

\section{Authorship Contributions}

Surgical and Medical Practices: F.N.K., F.K., S.K.G., Concept: E.E., F.K., S.D., H.U., S.K.G., Design: E.E., S.D., H.U., Data Collection or Processing: E.E., F.N.K., F.K., Analysis or Interpretation: E.E., S.D., H.U., S.K.G., Literature Search: E.E., F.N.K., F.K., S..K.G., Writing: E.E., H.U., S.K.G.

Conflict of Interest: No conflict of interest was declared by the authors.

Financial Disclosure: The authors declared that this study received no financial support.

\section{References}

1. Park SE. Epidemiology, virology, and clinical features of severe acute respiratory syndrome -coronavirus-2 (SARS-CoV-2; Coronavirus Disease-19). Clin Exp Pediatr. 2020;63:119-24.

2. Huang C, Wang Y, Li X, Ren L, Zhao J, Hu Y, et al. Clinical features of patients infected with 2019 novel coronavirus in Wuhan, China. Lancet. 2020;395:497506.

3. Obradović D, Esquinas AM. Hyponatremia at discharge: A solid risk or accidental findings in Community-acquired pneumonia. Eur J Intern Med. 2020;78:135-6

4. Nair V, Niederman MS, Masani N, Fishbane S. Hyponatremia in communityacquired pneumonia. Am J Nephrol. 2007;27:184-90.

5. Zilberberg MD, Exuzides A, Spalding J, Foreman A, Jones AG, Colby C, et al. Hyponatremia and hospital outcomes among patients with pneumonia: a retrospective cohort study. BMC Pulm Med. 2008;8:16.

6. Miyashita J, Shimada T, Hunter AJ, Kamiya T. Impact of hyponatremia and the syndrome of inappropriate antidiuresis on mortality in elderly patients with aspiration pneumonia. J Hosp Med. 2012;7:464-9.

7. Krüger S, Ewig S, Giersdorf S, Hartmann O, Frechen D, Rohde G, et al. Dysnatremia, vasopressin, atrial natriuretic peptide and mortality in patients with community-acquired pneumonia: results from the german competence network CAPNETZ. Respir Med. 2014;108:1696-705.

8. Schuetz P, Haubitz S, Christ-Crain M, Albrich WC, Zimmerli W, Mueller B; ProHOSP Study Group. Hyponatremia and anti-diuretic hormone in Legionnaires' disease. BMC Infect Dis. 2013;13:585

9. Yousaf Z, Al-Shokri SD, Al-Soub H, Mohamed MFH. COVID-19-associated SIADH: a clue in the times of pandemic! Am J Physiol Endocrinol Metab. 2020;318:E882-5

10. Frontera JA, Valdes E, Huang J, Lewis A, Lord AS, Zhou T, et al. Prevalence and Impact of Hyponatremia in Patients With Coronavirus Disease 2019 in New York City. Crit Care Med. 2020;48:e1211-7.

11. Habib MB, Sardar S, Sajid J. Acute symptomatic hyponatremia in setting of SIADH as an isolated presentation of COVID-19. IDCases. 2020;21:e00859.

12. Spasovski G, Vanholder R, Allolio B, Annane D, Ball S, Bichet D, et al. Clinical practice guideline on diagnosis and treatment of hyponatraemia. Eur J Endocrinol. 2014;170:G1-47.

13. Potasso L, Sailer CO, Blum CA, Cesana-Nigro N, Schuetz P, Mueller B, et al. Mild to moderate hyponatremia at discharge is associated with increased risk of recurrence in patients with community-acquired pneumonia. Eur J Intern Med. 2020;75:44-9,

14. Hawkins RC. Age and gender as risk factors for hyponatremia and hypernatremia. Clin Chim Acta. 2003;337:169-72.

15. Mannesse CK, Vondeling AM, van Marum RJ, van Solinge WW, Egberts TC, Jansen PA. Prevalence of hyponatremia on geriatric wards compared to other settings over four decades: a systematic review. Ageing Res Rev. 2013;12:16573

16. Nelson JM, Robinson MV. Hyponatremia in older adults presenting to the emergency department. Int Emerg Nurs. 2012;20:251-4.

17. Shapiro DS, Sonnenblick M, Galperin I, Melkonyan L, Munter G. Severe hyponatraemia in elderly hospitalized patients: prevalence, aetiology and outcome. Intern Med J. 2010;40:574-80.

18. Berl T. An elderly patient with chronic hyponatremia. Clin J Am Soc Nephrol. 2013;8:469-75 
19. Ellison DH, Berl T. Clinical practice. The syndrome of inappropriate antidiuresis. N Engl J Med. 2007;356:2064-72.

20. Koizumi K, Yamashita H. Influence of atrial stretch receptors on hypothalamic neurosecretory neurones. J Physiol. 1978;285:341-58.
21. Berni A, Malandrino D, Parenti G, Maggi M, Poggesi L, Peri A. Hyponatremia, IL-6, and SARS-CoV-2 (COVID-19) infection: may all fit together? J Endocrinol Invest. 2020;43:1137-9. 